\title{
Objective determination of skin phototypes in healthy children by diffuse reflectance spectroscopy
}

\author{
Petrovajova $\mathrm{M}^{1}$, Carska $\mathrm{N}^{1}$, Novotny $\mathrm{M}^{2,3}$ \\ Department of Pediatric Dermatovenerology, Children's University Hospital, Comenius University, \\ Bratislava, Slovakia. petrovajova.michaela@gmail.com
}

\begin{abstract}
Introduction: The clinical method of evaluating the color of the skin based on visual assessment is subjective and thus inaccurate. The objective determination of skin phototypes and levels of melanin in the skin is important for diagnosing the pigment disorders and also for adequate photoprotection. Diffuse reflectance spectroscopy (DRS) is a non-invasive, precise and objective method for quantifying the melanin levels.

Objective: The aim of this study is to assess the characteristic DRS spectrum of healthy skin in children and to detect the differences between them based on age, gender and skin phototype.

Material and methods: Skin pigmentation was measured by DRS in 73 children patients with experimental spectrophotometer UM-ES600. The amount of melanin is quantified from the obtained DRS spectra by proposed melanin quantification angle $\alpha$ obtained by comparing the reflectance properties of skin sample and universal depigmented sample (albino skin).

Results: We evaluated spectroscopic characteristics of children's healthy skin depending on age, gender and phototype. The value of melanin quantification angle a grew proportionally from phototype I to phototype IV, without any correlation to age or gender. We confirmed a clear association between clinical determination of Fitzpatrick's skin phototypes and objective data collected by DRS and related angle $\alpha$.

Conclusions: Our proposed index quantifies the difference in melanin levels in healthy children skin and also for different skin phototypes. The proposed method and melanin quantification angle a can further be used for the objectification of the progress of pigmentary diseases or for monitoring the effect of their therapy (Tab. 5, Fig. 4, Ref. 29). Text in PDF www.elis.sk.

Key words: diffuse reflectance spectroscopy, skin phototypes, quantification of skin melanin, melanin quantification angle $\alpha$.
\end{abstract}

The classification of humans into different sun-reactive skin types (I-VI) was introduced by Fitzpatrick (21) and has become a reference standard to assess the UV sensitivity of human skin. Fitzpatrick's skin types are divided into groups following the visual evaluation and history of sunburn and tanning. This condition is inaccurate mostly in children.

The visual clinical methods of the skin color evaluation for diagnostic purposes have been so far mostly subjective and thus inaccurate. Even when performed by a dermatologist, the outcome of visual assessment is influenced by effects such as those of light conditions, experience of the observer and human visual system conditions. The visual assessment is often limited to only a few values, leaving little room for quantifying subtle changes in melanin content. The melanin level assessment and skin phototype is important in clinical dermatology for exact diagnosis of pigmentary disorders and also for objective estimation of skin phototypes

${ }^{1}$ Department of Pediatric Dermatovenerology, Children's University Hospital, Comenius University, Bratislava, Slovakia, ${ }^{2}$ Faculty of Mathematics, Physics and Informatics, Comenius University, Bratislava, Slovakia, and ${ }^{3}$ Vis Gravis, s.r.o, Bratislava, Slovakia

Address for correspondence: M. Petrovajová, Department of Pediatric Dermatovenerology, Children's University Hospital, Limbová 1, SK-833 40 Bratislava, Slovakia.

Phone: +421.915383686 for adequate photoprotection. Therefore, objective investigative methods are needed for quantifying the melanin content. One of the objective investigated methods for skin color evaluation is the non-invasive diffuse reflectance spectroscopy (DRS). DRS measures the reflective properties of skin chromophores (melanin, hemoglobin) based on interaction with different wavelengths of visible light (400-750 nm). The outcome of DRS is a spectral curve expressing levels of reflectance response to different wavelengths. The interval of $620-720 \mathrm{~nm}$ of the spectra is significant for melanin response $(9,10,16,29)$.

Various experimental studies with DRS were performed to correlate skin type to different skin color parameters, e.g. the area under the intensity curve (AUIC) (13), slope and intercept of the spectrum in the range of 620-720 nm $(9,10,14,16,29)$, UV sensitivity in terms of minimal erythema dose (MED) $(22,23,24)$, or CIE L*a*b* color system $(1,14,25)$. Diffuse reflectance spectroscopy is a non-invasive, exact and objective investigation method for evaluating the skin color. However, the methods of DRS-data interpretation have so far not been fully developed.

To date, there has been no study on children's skin phototype evaluated with DRS. Therefore the objective of this study is to assess the characteristic DRS spectra of healthy children's skin and to detect differences in melanin levels based on age, gender and Fitzpatrick's skin phototype. Our proposed approach to 


\section{1-795}

DRS-data evaluation is easy to perform and suitable for everyday clinical practice.

\section{Material and methods}

\section{Human subject}

The skin pigmentation was measured by DRS method in 73 children (1 month - 18 years old) at the Department of Pediatric Dermatovenerology, Comenius University. The presented study analyzes the skin of Central European children. The skin type for this ethnic group varies generally between I -IV (64).

Human subjects were divided into groups by gender, age and Fitzpatrick scale. Tables 1-3 described the groups and the number of patients in each.

\section{Diffuse reflectance spectroscopy}

This study used the diffuse reflectance spectroscopy as a noninvasive and objective method to measure the skin pigmentation. DRS allows to acquire an objective quantification and comparison of melanin levels in the skin.

Skin pigmentation was measured by experimental spectrophotometer UM-ES600 $(28,29)$. The DRS setup consists of a light source, optic fiber $(500 \mu \mathrm{m})$, probe, analyzer and detection device. Measurements are conducted in standard conditions in dark room without daylight. Procedure is absolutely non-invasive while the probe of the spectrophotometer is in contact with the skin at the angle of $90^{\circ}$. No pressure on the probe was applied. Light is emitted from the light source and recorded 20 milliseconds later by the probe. The obtained signal is saved to the computer for analysis. Spectral signal is afterwards processed by special spectrophotometry software DermaSpec to form a spectral curve (28). For each patient, the averaged spectra from 3 different localities of normal skin of armpit were collected.

Tab. 1.

\begin{tabular}{lc}
\hline Gender & \\
\hline Group & Children \\
\hline Female & 41 \\
Male & 32 \\
\hline
\end{tabular}

Tab. 2.

\begin{tabular}{lc}
\hline Age & \\
\hline Group & Children \\
\hline$<1$ year & 12 \\
$1-5$ years & 10 \\
$6-10$ years & 15 \\
$11-15$ years & 16 \\
$15-18$ years & 20 \\
\hline
\end{tabular}

Tab. 3.

\begin{tabular}{lc}
\hline Fitzpatrick scale & \\
\hline Group & Children \\
\hline Phototype I & 15 \\
Phototype II & 32 \\
Phototype III & 19 \\
Phototype IV & 7 \\
\hline
\end{tabular}

The device is calibrated before the first measurement by measuring the reflectance spectrum of reflectance standard - the absolute white $S_{\text {ref }}(\lambda)$ ( $\mathrm{BaSO}_{4}$ powder). The calibration adjustment of the measured skin surface reflectance spectrum of the skin surface $\mathrm{S}(\lambda)$ is calculated using the raw measured spectrum $S_{\text {raw }}(\lambda)$, the $100 \%$ reflectance standard and dark reflectance standard $D(\lambda)(0 \%$ reflectance), where $\lambda$ represents wavelength $(2,17,28)$ :

$\mathrm{S}(\lambda)=\frac{\mathrm{S}_{\text {raw }}(\lambda)-D}{\mathrm{~S}_{\text {ref }}(\lambda)-D}$

The absorption spectrum $\mathrm{A}(\lambda)$ is defined as a negative decimal logarithm of the reflectance spectrum (14-17):

$A(\lambda)=-\log R(\lambda)$

The obtained spectral curves are further processed and visualized by the proprietary software visQuest developed for this purpose.

DRS-based melanin quantification angle $\alpha$ was calculated on curves obtained by comparing the reflectance properties of skin sample to universal depigmented sample (albino skin). Reflectance of albino skin sample seems to be a universal reference for spectroscopy for evaluation of melanin. The skin of albino patients provides exactly such a system where amelanotic skin has no structural differences from normal skin. By dividing the measured reflectance spectrum of the skin sample, we get a relative reflectance response $R(\lambda)$ of the skin. We then exploited the linear behavior of the spectral curve in the 620-720 nm interval significant for melanin response $(3,4,9,10,17,28)$, and we used the slope of the regression line to compute the melanin quantification angle $\alpha$. Starting with the regression line $\boldsymbol{y}=\boldsymbol{k} \boldsymbol{x}+\boldsymbol{q}$, where $k$ is the slope of the line and $q$ is the intercept, we scale the vertical dimension of the line by 1000 and calculate the horizontal angle of the line:

$\alpha=\arctan (k \cdot 1000) \cdot \frac{180}{\pi}$

The multiplication by 1000 and the conversion to degrees is arbitrary but it is important for human readability of the results which otherwise would include hard-to-discern small fractional numbers.

\section{Results}

Using diffuse reflectance spectroscopy, we obtained 73 reflectance spectra measured in standard conditions on the non-exposed healthy skin on the inside part of the right shoulder near axilla. Reflectance spectra of all children are shown in Figure 1. Red curves depict girls, blue curves depict boys. The reflectance curve marked in yellow expresses the average reflectance of all the children.

Axilla (armpit) from the inner side of the upper arm was selected as the default part of the body for the spectrophotometric evaluation of constitutive skin pigmentation, presented as standard also by other authors $(4,7,10,14,15,17,29)$. This part of the body seems to be most appropriate due to the minimum load rating sunlight since this part of the body is usually not exposed to the sun.

The difference is visually not obvious (Graph 1) and the measured reflectance shows how the reflectance curve changes its shape. 


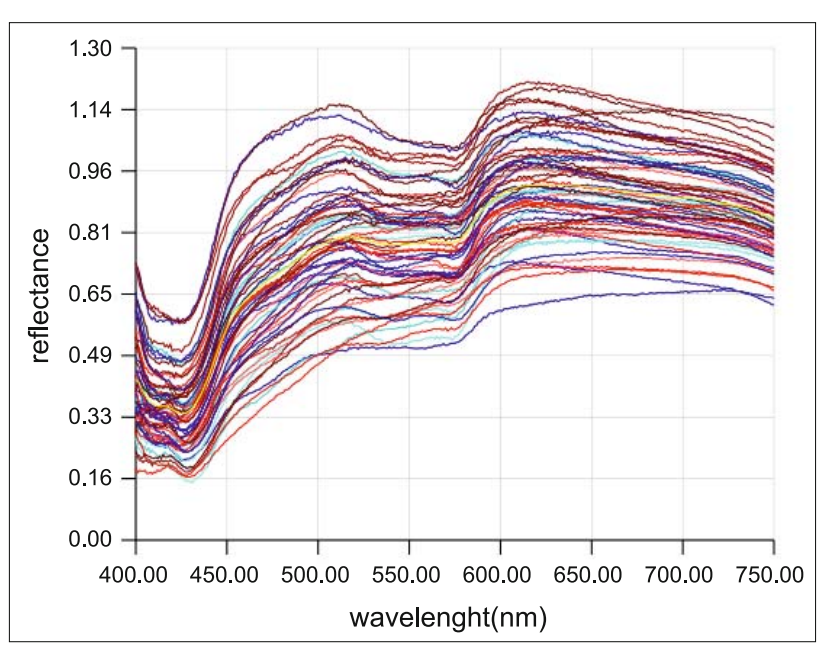

Fig. 1. The reflectancies of healthy skin of all patients: blue spectras mean boys, red spectras mean girls, light tints reflect young age, darkest ones mean the oldest age group. The spectral curve of yellow color means the average reflectance of all 73 patients.

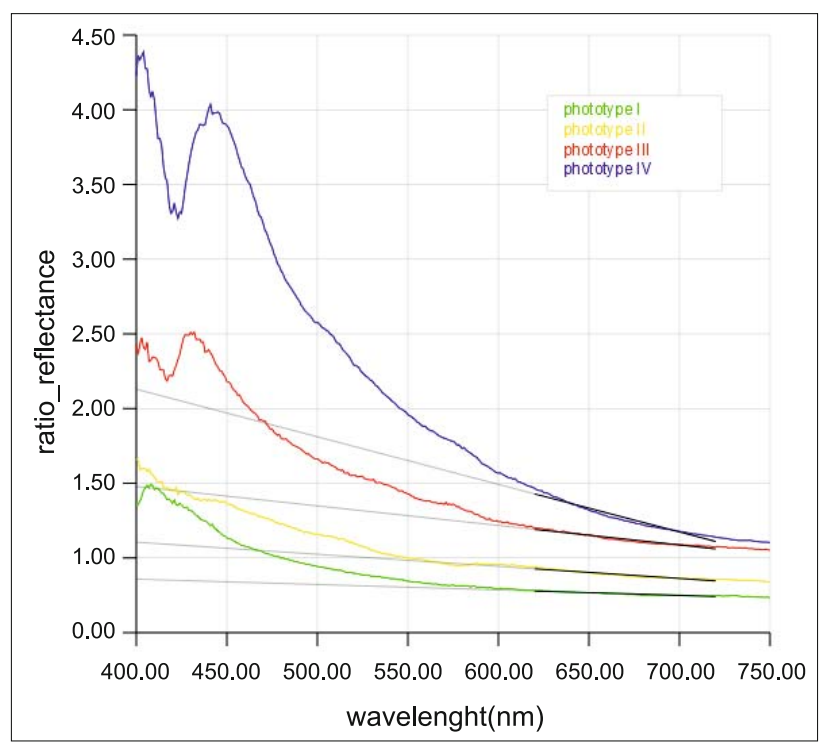

Fig. 2. The evaluation of skin melanin by melanin quantification angle $\alpha$ of four patient with different phototype I-IV. The short black straith lines mean a linear regressions in the range of wavelenghts 620-720 $\mathrm{nm}$, with displayed angle $\alpha$.

The absence of any pattern regarding age or gender shows that the reflectance values of all patients are affected neither by gender nor by age. All the spectras of healthy skin have similar shapes, trends and characteristics with minimal differences between age and gender groups. Specific spectral curves do show certain differences among the patients but these are partially due to measurement noise (jagged curves) and partially due to constant translation of a whole curve upward or downward. Neither of these two effects are significant for our further process.

For mutual comparison and objectification of constitutive pigmentation of healthy skin in the population of healthy children, we used a sample calculation of proposed melanin quantification angle $\alpha$. The slope of spectral curve (part $620-720 \mathrm{~nm}$ ) is conditional to the amount of melanin in the skin which is responsible for its constitutial pigmentation. The evaluation of skin melanin of each phototype (I-IV) is shown in Figure 2. The values of melanin quantification angle $\alpha$ of all patients $(n=73)$ in consideration of age, gender and phototypes groups are shown in the Table 4.

We used the statistical methods in Matlab software to evaluate the significance of the acquired results. According to Jarque-Bera test, all of our tested populations showed normal distribution and are suitable for evaluation. The differences in $\alpha$ between genders were analyzed by unpaired Student t-test and displayed no significance. The differences in angle $\alpha$ between age groups were estimated by the one-factor analysis of variation (one-way ANOVA) and Bonferroni post-hoc test, again with no significance. ANOVA was also used for differences in $\alpha$ between phototype groups. This proved significant and the correlation of quantification angles $\alpha$ and different phototypes is depicted by a boxplot in Figure 3 and as Kendal serial correlation coefficient tau-b in Figure 4. The results of analysis are summarized in Table 5.

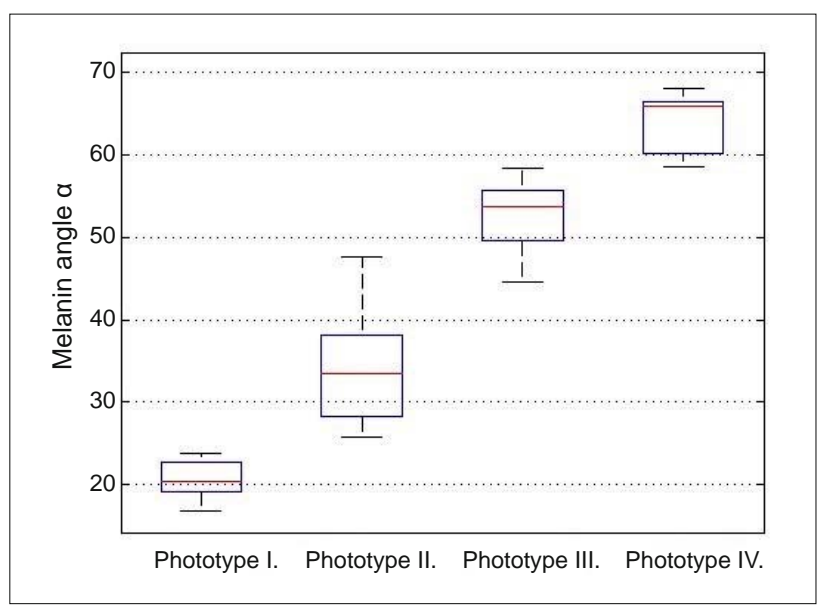

Fig. 3. Phototype groups I-IV of healthy children $(n=73)$ based on values of melanin quantification angle $\alpha$.

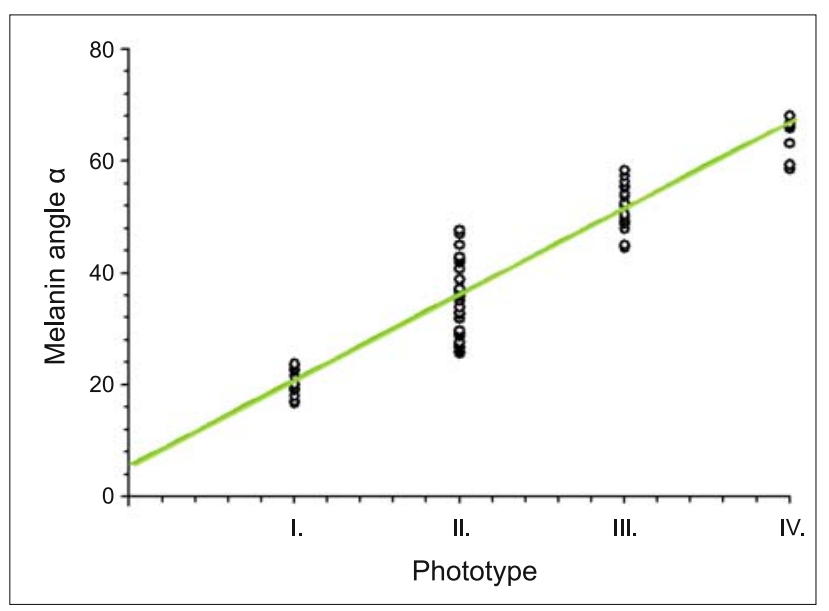

Fig. 4. Correlation of phototype I-IV and melanin quatification angle $\alpha$ of healthy children $(n=73)$, correlation coefficient of melanin growth tau $b=0.83$. 
Tab. 4. The melanin quantification angle $\alpha$ in children with healthy skin $(n=73)$ divided into groups by age, gender and phototype.

\begin{tabular}{|c|c|c|c|c|c|c|c|c|c|}
\hline \multicolumn{2}{|c|}{ Melanin angle $\alpha$} & \multicolumn{2}{|c|}{ Phototype I } & \multicolumn{2}{|c|}{ Phototype II } & \multicolumn{2}{|c|}{ Phototype III } & \multicolumn{2}{|c|}{ Phototype IV } \\
\hline & & Male & Female & Male & Female & Male & Female & Male & Female \\
\hline \multirow{5}{*}{ 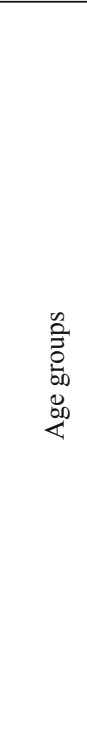 } & $<1$ & 22.60 & 23.56 & 28.80 & $\begin{array}{l}29.23 \\
35.22 \\
47.05 \\
26.60\end{array}$ & $\begin{array}{l}45.01 \\
55.60 \\
49.39 \\
53.68 \\
49.99\end{array}$ & & & \\
\hline & $1-5$ & & 22.80 & $\begin{array}{l}41.95 \\
32.94\end{array}$ & 33.85 & $\begin{array}{l}52.56 \\
55.85\end{array}$ & $\begin{array}{l}52.00 \\
48.78 \\
56.36\end{array}$ & & 59.27 \\
\hline & $6-10$ & 19.49 & $\begin{array}{l}23.46 \\
23.78\end{array}$ & $\begin{array}{l}36.97 \\
38.92\end{array}$ & $\begin{array}{l}33.84 \\
42.51 \\
25.88 \\
37.25\end{array}$ & 54.21 & $\begin{array}{l}53.69 \\
48.03\end{array}$ & 63.26 & $\begin{array}{l}58.66 \\
66.62\end{array}$ \\
\hline & $11-15$ & $\begin{array}{l}20.27 \\
21.63 \\
19.91\end{array}$ & $\begin{array}{l}19.40 \\
19.94\end{array}$ & $\begin{array}{l}26.60 \\
31.87 \\
29.69 \\
47.70 \\
40.85 \\
\end{array}$ & $\begin{array}{l}35.89 \\
42.97 \\
31.83 \\
29.28\end{array}$ & & 57.52 & 68.11 & \\
\hline & $16-18$ & & $\begin{array}{l}16.81 \\
18.16 \\
22.51 \\
17.26\end{array}$ & $\begin{array}{l}36.16 \\
27.79 \\
27.59 \\
45.03\end{array}$ & $\begin{array}{l}32.65 \\
25.77 \\
27.08 \\
36.80 \\
25.96\end{array}$ & $\begin{array}{l}55.39 \\
58.45\end{array}$ & $\begin{array}{l}58.24 \\
50.47 \\
44.63\end{array}$ & 66.20 & 65.97 \\
\hline \multicolumn{2}{|c|}{ Number of patients: } & \multicolumn{2}{|c|}{15} & \multicolumn{2}{|c|}{32} & \multicolumn{2}{|c|}{19} & \multicolumn{2}{|c|}{7} \\
\hline
\end{tabular}

Tab. 5. Statistical comparison of groups according to age, gender and skin phototype.

\begin{tabular}{lccc}
\hline & Age groups & Gender & Phototype \\
\hline Statistical method & one-way ANOVA & non-paired t-test & one-way ANOVA \\
Significance level & $\mathrm{p}=0.5131$ & $\mathrm{p}=0.2348$ & $\mathrm{p}<0.001$ \\
Outcome & non-significant (all groups) & non-significant & significant (all groups) \\
\hline
\end{tabular}

The interpretation of the statistical test's results:

- Without statistical significance of different parametres of melanin angles $\alpha$ between each age groups $(\mathrm{p}=0.5131)$.

- Without statistical significance of different parametres of melanin angles $\alpha$ between male and female $(p=0.2348)$

- Statistical significance of different parametres of melanin angles $\alpha$ between groups divided by phototypes $(\mathrm{p}<0.001)$.

Considering the results of this statistical evaluation, we can state that the values of melanin quantification angle $\alpha$ in the group of healthy children are unrelated to gender or age. This confirms a definition of constitutive skin pigmentation in the clinical practice as given genetically and not being dependent on age or gender. On the other hand, the values of $\alpha$ are tightly related to groups based on skin phototype (Figs 3 and 4). There is a strong positive linear relationship (correlation coefficient) between increasing values of melanin quantification angle $\alpha$ and phototype (tau $b=0.83(95 \%$ interval of confidence 0.77 to 0,89$)$ ). The skin of higher phototype number contains a higher level of melanin.

In summary, we can propose that the value of melanin quantification angle $\alpha$ obtained from the evaluation of spectrophotometric measurements by DRS shows the amount of melanin in measured samples. We can use this quantification angle $\alpha$ as an objective parameter in the evaluation of the skin pigmentation. Our proposed angle calculation expresses the extent of the difference in melanin levels in healthy skin of children. We successfully used this quantification angle $\alpha$ to quantify the variations in skin melanin in different skin phototypes. The melanin quantification angle $\alpha$ is a reliable parameter for evaluating the skin melanin amount in vivo. Owing to this value, the melanin levels can be easily and objectively compared between different individuals as well as in different stages of diseases or during therapy monitoring.

\section{Discussion}

In our work we elaborate and evaluate the characteristics of spectral curves of healthy skin via their changes in shapes in group of 73 children depending on age, gender and phototype. To date, there has been no known study conducted on children population. Nevertheless, the acquisition of diffuse reflectance spectrophotometry in determination of skin phototypes was confirmed by other authors $(4,7,13,14,29)$.

Our group of population comprised healthy children from one month to 18 years of age with clinically determined phototypes I-IV by Fitzpatrick. DRS measurements were conducted on the patients in standard conditions on the inner side of the arm, in the axila. The reflectance spectral curves from different samples of healthy skin displayed slight differences but they share similar shape and trend which is typical for the most common phototypes 
II and III. We based the evaluation of the differences between the groups on measured spectral curves compared to universal amelanotic standard (albino skin) for each patient and thus established the melanin quantification angle $\alpha$.

The differences in $\alpha$ values proved to be statistically significant among groups based on phototype. Groups based on gender or age showed no relationship to $\alpha$ values. Depending on amount of predisposed melanin in the skin, the phototype is determined by genetic pigmented constitution of the skin and stays the same during the whole life. The constitutive skin pigmentation does not change over time, it is an innate predisposition of each individual $(26,27)$. The statistically significant relationship between the proposed melanin quantification angle $\alpha$ and the level of phototype displays a positive correlation. Thus, the melanin quantification angle $\alpha$ is a reliable parameter for objective determination of melanin amount in the skin. The objective determination of phototype is very important for clinical purposes, e.g. before laser therapy, in differential diagnosis in different diseases and for determining the best photoprotection $(7,14,27,29)$.

\section{Conclusion}

DRS and the melanin quantification angle $\alpha$ were used to evaluate spectroscopic characteristics of healthy skin of children depending on age, gender and phototype. The melanin angle $\alpha$ value grew proportionally from phototype I to phototype IV without correlation to age or gender of the children. We confirmed a clear association between clinical visual inspection of Fitzpatrick skin types and objective data collected by DRS (melanin quantification angle $\alpha$ ).

We presented a detailed description of novel approach to DRS melanin evaluation. DRS melanin evaluation is a reliable investigation method for quantifying pigmentation in clinical practice. This method is non-invasive, exact and objective and it is much more sensitive to subtle changes in skin pigmentation as compared to personal visual inspection. It is supposed to be a suitable method for further measures of skin color in pigment disorders and for therapy monitoring mainly in pediatric patients.

\section{References}

1. Alghamdi KM, Kumar A, Taieb A, Ezzedine K. Assessment methods for the evaluating of vitiligo. JEADV 2012; 26 (12): 1463-1471.

2. Anderson RR, Parish AJ. The optics of human skin. J Invest Dermatol 1981; 77: 13-19

3. Dolotov LE, Sinichkin YuP, Tuchin VV, Utz SR, Altshuler GB, Yaroslavsky IV. Design and evaluation of a novel portable ertyhema-melanin-meter. Lasers Surg Med 2004; 34: 127-135.

4. González FJ, Martinéz-Escanamé M, Munoz RI, Torres-Árvalez B, Moncada B. Diffuse reflectance spectrophotometry for skin phototype determination. Skin Res Technol 2010; 16: 397-400.

5. Hamzavi I, Shiff N, Martinka M, Huang Z, McLean D, Zeng H, Lui H. Spectroscopic assessment of dermal melanin using blue vitiligo as in vivo model. Photodermatol Photoimmunol Photomed 2006, 22: 46-51.

6. Hegyi V, Hegyi J. Methods and Agents for Pigmentation and Depigmentation - Sense or Nonsense? Hautarzt 2010; 61 (7): 586-592.

7. Karsten AE, Singh A, Karsten PA, Braun MWH. Diffuse reflectance spectroscopy as a tool to measure the absorbtion coefficient in skin: South African skin phototypes. Photochem Photobiol 2013; 89: 227-233.
8. King RA, Summers CG. Albinism: Ocular and Oculocutaneous Albinism and Hermansky-Pudlak Syndrome. Manag Genet Syndr 2010; 53-68.

9. Kollias N, Baqer A. Spectroscopic characteristic of human melanin in vivo. J Invest Dermatol 1985; 85: 38-42.

10. Kollias N, Baqer A. On the assessment of melanin in human skin in vivo. Photochem Photobiol 1986; 34: 49-54.

11. Kollias N, Stamatas GN. Optical Non-invasive approaches to diagnosis of skin diseases. J Invest Dermatol Symp Proc 2002; 7: 64-75.

12. Latreille J, Gardinier S, Ambroisine L, Mauger E, Tenenhaus M, Guehenneux S, Morizot F, Tschachler E, Guinot Ch. Influence of skin colour on the detection of cutaneous erythema and tanning phenomena using reflectance spectrophotometry. Skin Res Technol 2007; 13: 236-241.

13. Pershing LK, Tirumala VP, Nelson L, Corlett JL, Lin AG, Meyer LJ, Leachman SA. Reflectance spectrophotometer: The dermatologists sphygmomanometer for skin phototyping? J Invest Dermatol 2008, 128: 1633-1640.

14. Ries F, Kapoustina V, Kron A, Fink A, Radle M. Estimation of skin phototypes with optical parameters: an experimental study using newly developed fibre optic detection device. Intern J Cosm Sci 2013; 35: 50-56.

15. Stamatas GN, Wu J, Kollias N. Non-invasive method for quantitative evaluation of exogenous compound deposition on skin. J Invest Dermatol 2002; 118 (2): 295-302.

16. Stamatas GN, Zmudzka BZ, Kollias N, Beer JZ. Non-Invasive measurement of skin pigmentation in situ. Pigment Cell Res 2004; 17: 618-626.

17. Stamatas GN,Zmudzka BZ, Kollias N, Bear JZ. In vivo measurement of skin erythema and pigmentation: new means of implementation of diffuse reflectance spectroscopy with a commercial instrument. Br J Dermatol 2008; 159: 683-690.

18. Taieb A, Picardo M. The definition and assessment of vitiligo: a consensus report of the Vitiligo European Task Force. Pigment Cell Res 2007; 20: 27-35.

19. Wagner JK, Jovel C, Norton L, Parra EJ, Shriver MD. Comparing quantitative measures of erythema, pigmentation and skin response using reflectometry. Pigment Cell Res 2002; 15: 379-384.

20. Zonios G, Bykowski J, Kollias N. Skin melanin, hemoglobin, and light scattering properties can be quantitatively assessed in vivo using diffuse reflectance spectroscopy. J Invest Dermatol 2001; 117: 1452-1457.

21. Fitzpatrick TB. The validity and practicality of photo-reactive skin types 1 though 6. Arch Dermatol 1988; 124: 869-871.

22. Westerhof W, Estevez-Uscanga O, Meens J, Kammeyer A, Durocq M, Cario I. The realation between constitutional skin color and photosensitivity estimated from UV-induced erythema and pigmentation dose-curve response curves. J Invest Dermatol 1990; 94: 812-816.

23. Palmer R, Aquilina S, Milligan PJ, Walker SI, Hawk LM, Yuong AR.. Photoadapting during narrowband ultraviolet-b therapy is independent of skin type: a study of 352 patients. J Invest Dermatol 2006; 26: 1256-1263.

24. Lee JH, Kim TY. Relationship between constitutive skin color and ultraviolet light sensitivity in Koreans. Photodermatol Photoimmunol Photomed 1999; 15: 231-235.

25. Taikiwaki H. Measurement of skin color_Practical application and theoretical considerations. J Med Invest 998; 44: $121-126$

26. Park JH, Lee MH. A study of skin color by melanin index according to site, gestational age, birth weight and season of birth in korean neonates. J Korean Med Sci 2005; 20: 105-108.

27. Tsumura N, Kawabuchi M, Haneishi H, Miyake Y. Mapping pigmentation in human skin by multi- visible- spectral imaging by inverse optical scattering technique. J Imaging Sci Technol 2001; 45: 444-450.

28. Soršáková Trnovská K, Keppert M, Hegyi V. Diffuse Reflectance Spectrophotometry I. Spectrophotometer and its engineering solution. Čes Slov Derm 2009; 84: 109-115.

29. Soršáková Trnovská K, Hegyi V. Diffuse Reflectance Spectrophotometry II. Practical Applications - a pilot study. Čes Slov Derm 2010; 85: 21-28.

Received May 13, 2013. Accepted February 20, 2014. 